In: Itoh S., Shukla S. (eds) Advanced Surface Enhancement. INCASE 2019. Lecture Notes in Mechanical Engineering. Springer, Singapore https://doi.org/10.1007/978-981-15-0054-1_15

\title{
Bonding Strength Improvement through Numerical Simulation of Particle Impact Process during Metal Cold Spray
}

\author{
Song $\mathrm{X}^{1,5}$, Jin $\mathrm{XZ}^{2}$, Everaerts $\mathrm{J}^{3}$, Tan $\mathrm{WY}^{2}$, Sun $\mathrm{W}^{2}$, Marinescu $\mathrm{I}^{4}$, Zhai $\mathrm{W}^{5}$, Li F ${ }^{4}$, Liu \\ $\mathrm{EJ}^{2}$, Korsunsky $\mathrm{AM}^{3}$ \\ ${ }^{1}$ School of Mechanical and Automation Engineering, Chinese University of Hong Kong, \\ William M.W. Mong Engineering Building, Hong Kong \\ ${ }^{2}$ School of Mechanical and Aerospace Engineering, Nanyang Technological University, 50 \\ Nanyang Avenue, Singapore 639798 \\ ${ }^{3}$ Department of Engineering Science, University of Oxford, Oxford, UK OX1 3PJ \\ ${ }^{4}$ Rolls-Royce Singapore Pte Ltd, 1 Seletar Aerospace Crescent, Singapore 797565 \\ ${ }^{5}$ Singapore Institute of Manufacturing Technology, Agency for Science, Technology and Re- \\ search (A*STAR), Singapore 637662
}

\begin{abstract}
Cold spray is an emerging additive manufacturing technique with potential applications in surface functionalization, bulk component production and restoration/repair. During the cold spray process, metallic powders are accelerated to supersonic velocities by the carrier gas of high pressure and temperature and impact on the substrate to form layers of coating through deformation-induced bonding. However, the coating fabricated by this process suffers from low cohesive strength and weak interfacial bonding. Therefore, process optimization through numerical simulation is much needed. Here we employ finite element simulation with Johnson-Cook plasticity and dynamic failure model to numerically predict the temperature distribution within single particle, and they show good agreement with experimental observation using SEM. This provides a validated description of microscopic phenomena using numerical simulation, hence it can be employed further to study the bonding strength of the metal cold spray coating. Through microstructural analysis, we propose a semi-empirical relationship between the nodal temperature profile and local bonding strength, hence identified that the increase of the localized bonding area in a single splat is the determining factor for the increase of the bonding strength.
\end{abstract}

Keywords: Metal Cold Spray, Finite Element Simulation, Interfacial Bonding Strength.

\section{Introduction}

Metal Cold Spray (MCS) is an emerging additive manufacturing technology with great potential in coating and repair. The solid powders are accelerated to supersonic velocities by the carrier gas of high pressure and temperature created from the convergent- 
divergent nozzle, and hit on the prepared substrate to form layers of coating through intensive plastic deformation. One advantage of the cold spray technique is to keep the powder and substrate temperatures below the melting point so that the initial physical and chemical properties of the material can be retained. The metallic bonding formed at the particle/substrate interface in cold spraying process is attributed to "adiabatic shear instability", which was proposed by Assadi et al. [1]. Furthermore, when the particle is impacting on the substrate at a velocity above the critical velocity, the contact process is so quick that the heat generated by the plastic deformation greatly increases the local temperature and softens the material. As a result of the thermal softening, the rate of strain hardening is reduced while the strain and the temperature are abruptly increased at that region. This secondary jump in temperature and strain (as compared with the initial temperature jump due to particle's first contact with the substrate, which is universal across all particle contact areas) can be obtained using finite element analysis, and it is one of the unique features of the metal cold spray process. In this work, we attempt to quantitatively link this characteristics with the interfacial bonding strength, which provides a valuable predictive tool for the process engineers to carry out virtual parametric study.

\section{Methodology}

\subsection{Finite Element Simulation}

The method of constructing the finite element (FE) model used in this paper has been described in detail in previous work [2]. It is provided briefly here again for completeness.

Taking into account the experimental configuration, a three dimensional FE model has been developed with ABAQUS/Explicit to simulate the impact between a single sprayed power particle and the substrate (Fig. 1). Accordingly, 8-node hexahedral elements with reduced integration and stiffness hourglass control (C3D8R) were employed with meshing size gradually increases from $0.3 \mu \mathrm{m}$ at the impact center to $1.0 \mu \mathrm{m}$ at rest of the part. The diameter of the spherical particle is set to be $30 \mu \mathrm{m}$ which is taken from the average diameter of the powder particle used in the experiments. The Johnson - Cook plasticity and dynamic failure model is applied to simulate the plastic deformation, strain hardening and progressive damage of both the particle and substrate. The material properties and the parameters of the Johnson - Cook model for Ti-6Al-4V are applied to both the particle and the substrate in the FE model according to previous literature [3].

Up to this point, the bonding at the contact surface has not been well defined in the model. The particle is still allowed to bounce back which makes the simulation results invalid after the separation. Therefore, the normal behaviour of the interaction property has to be set in a way that there is no separation or penetration allowed at the interface during the whole impact process. The more precise but complicated and laborious way to define the bonding is to employ the user-defined subroutine. At this stage of study,

Song X. et al. (2020) Bonding Strength Improvement ..., In: Itoh S., Shukla S. (eds) Advanced Surface Enhancement. INCASE 2019. Lecture Notes in Mechanical Engineering. Springer, Singapore

https://doi.org/10.1007/978-981-15-0054-1_15 
a much more simplified but robust way can be found in ABAQUS/Explicit, which uses the over-closure dependent pressure to tie the two contact surfaces together. The details of the numerical settings are provided in the literature [2] as well.

Although using animation of the impact process can show no separation or penetration at the contact surface. The effect of using "softened" contact has to be evaluated quantitatively to validate this new model. We employ the velocity of the top node on the particle as an indicator and plot it against time in Fig. 2 for two different contact models. The two velocity trends share the same path before decreasing to 0 . After the particle tends to bounce back, the non-separation constraint in "softened" contact model "pulls" the particle back and the node velocity returns and stays at 0, as shown in Fig 2 .

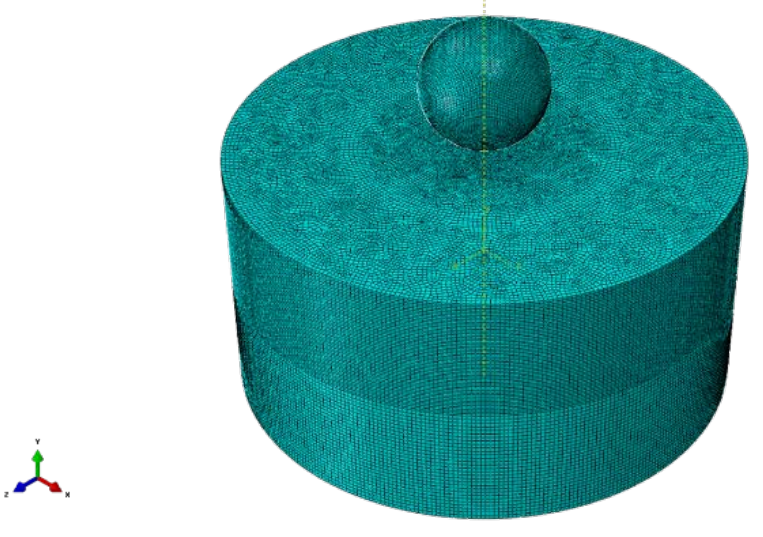

Song X. et al. (2020) Bonding Strength Improvement ..., In: Itoh S., Shukla S. (eds) Advanced Surface Enhancement. INCASE 2019. Lecture Notes in Mechanical Engineering. Springer, Singapore

https://doi.org/10.1007/978-981-15-0054-1_15 
Fig. 1. 3D model for metal cold spray single particle impact (normal).

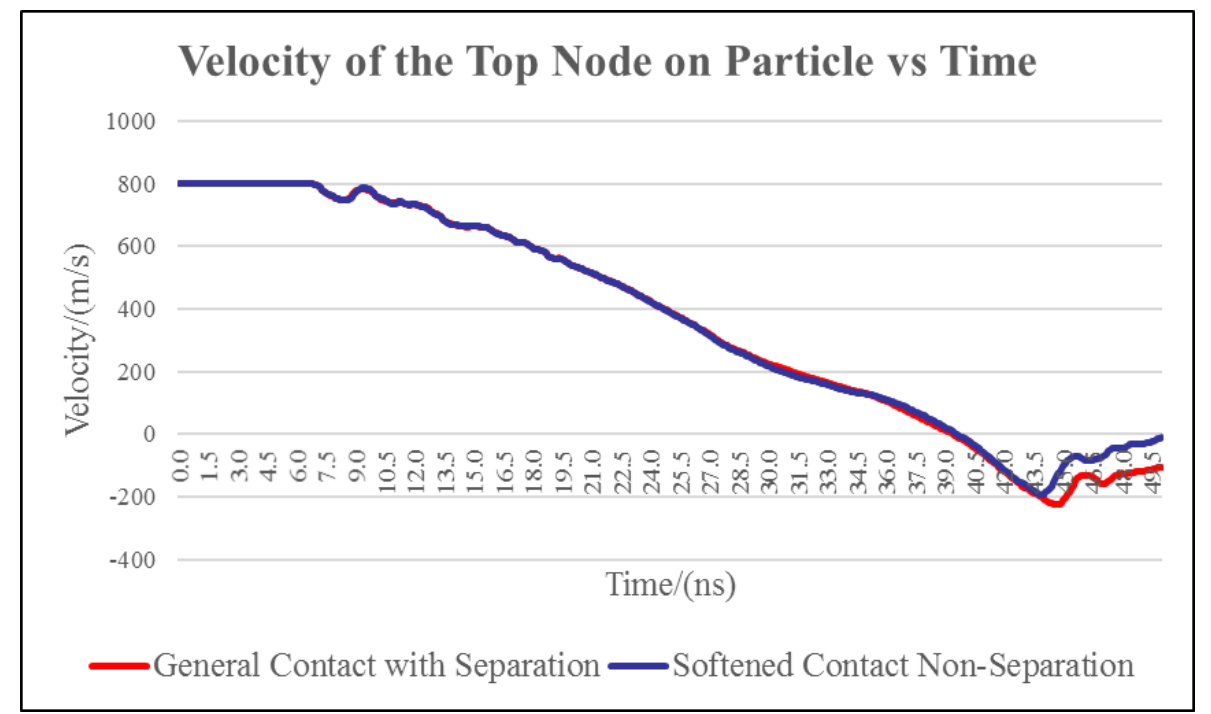

Fig. 2. Velocity trends of the top node on particle for models for two different contact models.

\subsection{Experimental setup}

Ti-6Al-4V Grade 23 powder with average particle size of $30 \mu \mathrm{m}$ was employed as the feedstock and the substrate was Ti-6Al-4V Grade 5 block with mirror-like top surface finish. The experimental setup follows previous work [4] with powder feed rate $30 \mathrm{~g} / \mathrm{min}$ and transverse scan speed $500 \mathrm{~mm} / \mathrm{s}$. After the spray, mini-tensile test coupons were cut out from the block, which is illustrated in Fig. 3. Following the work of Tan WY [5], mini-tensile tests were carried out by pulling the samples with in-house designed jig until sudden rupture occurred at the interface. The UTS (Ultimate Tensile Strength) can be obtained from this exercise by dividing the maximum load with the nominal cross section (as for this brittle interface). The fracture surfaces were further examined by SEM (JSM-IT300, JEOL Ltd., Tokyo, Japan) to identify the particles of interest.

Song X. et al. (2020) Bonding Strength Improvement ..., In: Itoh S., Shukla S. (eds) Advanced Surface Enhancement. INCASE 2019. Lecture Notes in Mechanical Engineering. Springer, Singapore

https://doi.org/10.1007/978-981-15-0054-1_15 

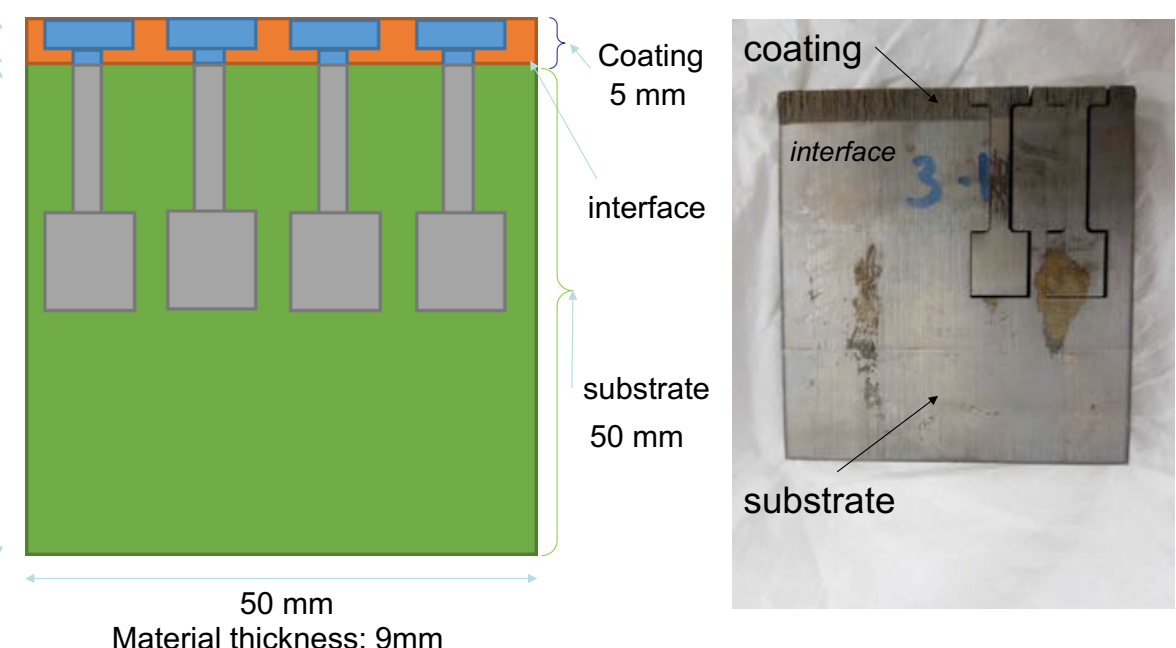

Fig. 3. Mini-tensile test dog-bone samples preparation

\section{Results and Discussion}

\subsection{Single particle impact model validation}

Since the fraction of the bonding nodes for the two different contact models are distinct from each other, it is necessary to compare the results with the surface morphology of the single particle splat in the coating. As compared in Fig. 4, the temperature contours of the bottom surface on the deformed particle shows high similarity with the surface morphology of the single particle in the coating fracture surface. The large outer ring area of the particle is identified as the bonding region, which corresponds to the high temperature region in the simulation. Conversely, the center region of the particle bottom is very smooth, showing no sign of bonding. This corresponds to the low temperature region in the simulation, which is of the same size as in the SEM. This comparison further reinforces the validity of the model using the Johnson - Cook plasticity and damage model and non-separation constraints to bond the particle and substrate together.

Song X. et al. (2020) Bonding Strength Improvement ..., In: Itoh S., Shukla S. (eds) Advanced Surface Enhancement. INCASE 2019. Lecture Notes in Mechanical Engineering. Springer, Singapore

https://doi.org/10.1007/978-981-15-0054-1_15 

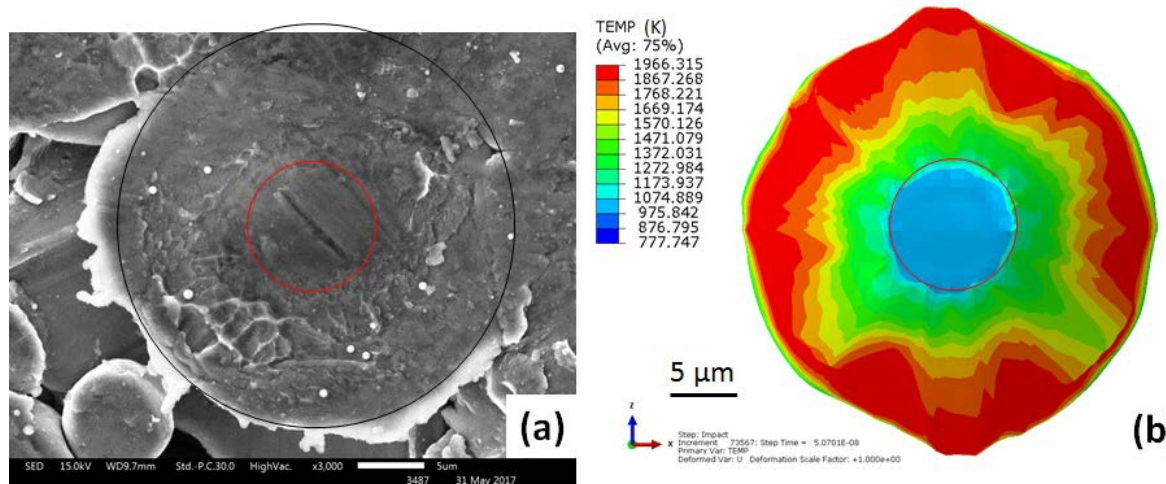

Fig. 4. Comparison between simulated temperature contour and surface morphology at the bottom of the deformed particle.

\subsection{Derivation of the bonding stress from the nodal temperature profile}

The additional/second temperature jump which indicates the onset of adiabatic shear instabilities and bonding is termed as "secondary jump", as there will always be a temperature jump at the initial stage when the particle land on the substrate at the beginning of the impact process. In a temperature history plot, "secondary jump" is characterized by the second obvious increase of the slope of the temperature history line, which is circled in red in Fig. 5. Fig. 5 provides an example of the temperature profiles of individual bonding nodes due to adiabatic shear instability. Although many previous studies have suggested the link between the phenomenon of temperature secondary jump and the surface bonding in cold spray $[1,6,7]$, none of them have actually provided this correlation numerically. In this work, a numerical model is developed below with a few assumptions.

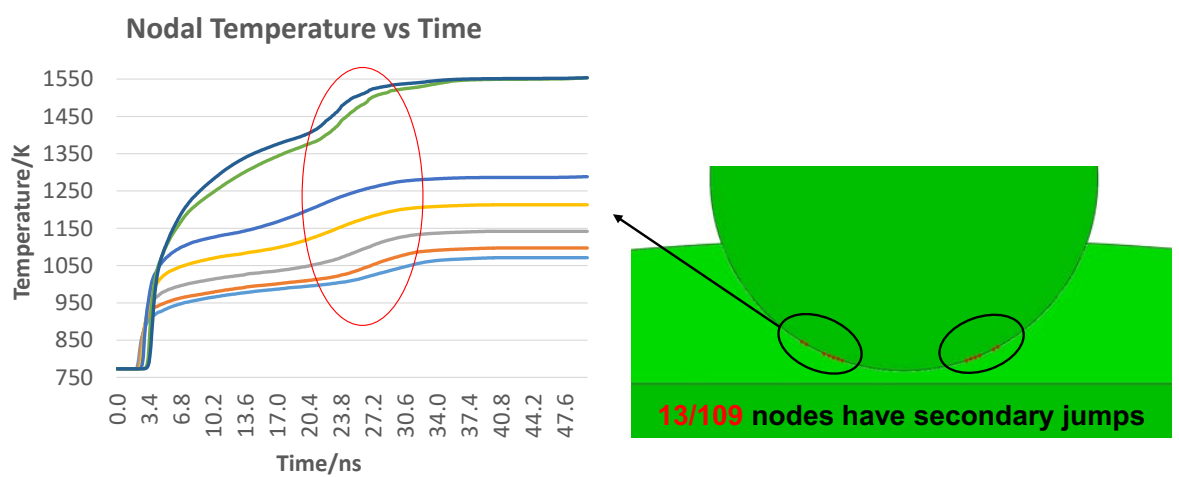

Fig. 5. Temperature profiles of the individual nodes with adiabatic shear instability and highlight of their locations in the particle.

Song X. et al. (2020) Bonding Strength Improvement ..., In: Itoh S., Shukla S. (eds) Advanced Surface Enhancement. INCASE 2019. Lecture Notes in Mechanical Engineering. Springer, Singapore

https://doi.org/10.1007/978-981-15-0054-1_15 
First, the rate of temperature change $T^{\prime}$ is the local slope of the temperature history plot. The onset of the "jump" phenomenon corresponds to the change of the local slope, which can be expressed as derivative of the slope $\left(T^{\prime}\right)^{\prime}$, and it is equal to the second derivative of temperature over time T". Therefore, with this approach, the "secondary jump", which is the sudden increase of temperature rising rate, should be sensed automatically by detecting a big value of $T$ "'. This is to identify the nodes that have bonding in the model automatically using one single value. Moreover, we can indirect validate the proposed criterion by running the model at different impact speeds and plot the $T^{\prime \prime}$ contour, as shown in Fig. 6. It can be seen that with increasing speed, the bonding area within one particle increases accordingly. It can be validated by the SEM analysis of the fracture surface of the residual of a single particle splat on the substrate. Fig. 7 highlights different bonding areas in single particle splat with different impact speeds. The donut rings cover the bonding area of a single particle splat with characteristic rough fracture surface. The area percentage increases with the increasing speed as well. The center of the particle is still smooth, indicating no bonding at that area, also same as in the Fig. 6. In this way, we can state that the increase of the localized bonding area is the determining factor for the increase of the bonding strength.
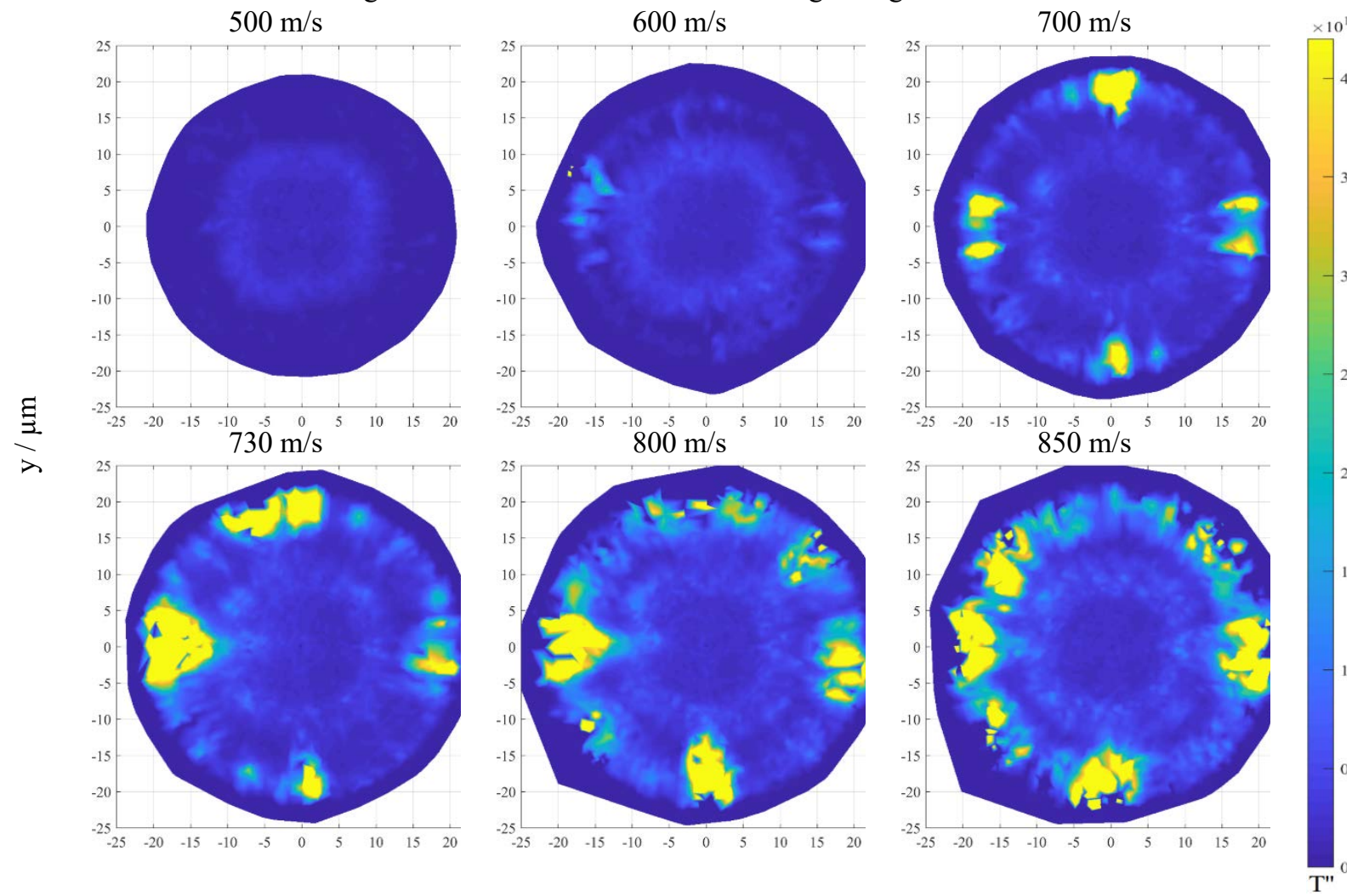

Song X. et al. (2020) Bonding Strength Improvement ..., In: Itoh S., Shukla S. (eds) Advanced Surface Enhancement. INCASE 2019. Lecture Notes in Mechanical Engineering. Springer, Singapore

https://doi.org/10.1007/978-981-15-0054-1_15 
$\mathrm{x} / \mu \mathrm{m}$

Fig. 6. Contour plots of the simulated $\boldsymbol{T}^{\prime \prime}$ values in the contact surface at different speeds

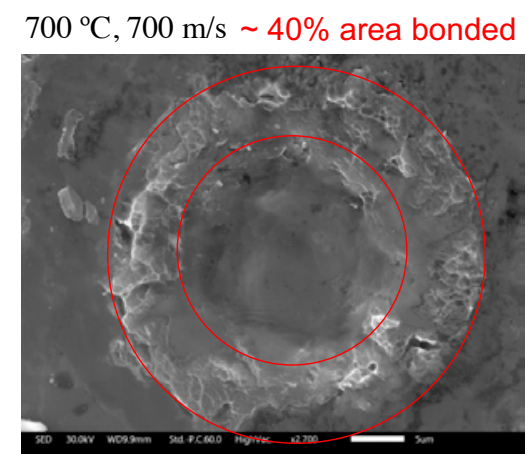

$900{ }^{\circ} \mathrm{C}, 760 \mathrm{~m} / \mathrm{s} \sim 70 \%$ area bonded

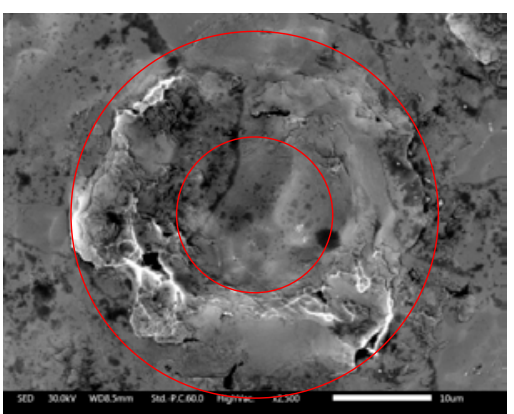

$800{ }^{\circ} \mathrm{C}, 730 \mathrm{~m} / \mathrm{s} \sim 60 \%$ area bonded

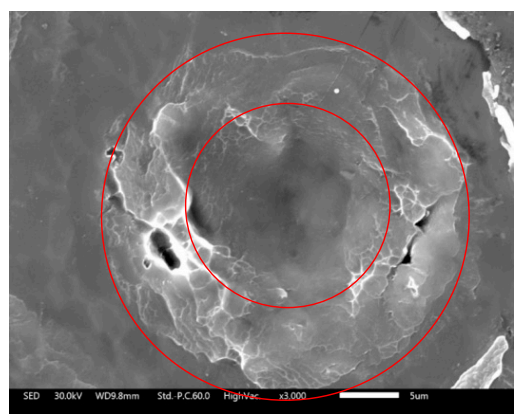

$1000{ }^{\circ} \mathrm{C}, 800 \mathrm{~m} / \mathrm{s} \sim 80 \%$ area bonded

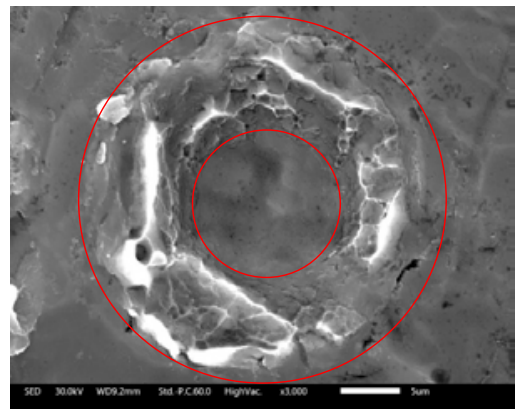

Substrate - Magnification $\sim 3000 \times$

Fig. 7. the SEM images of fracture surfaces of a single particle at different impact speeds

Furthermore, for those bonded nodes, the bonding strength should, naturally, be determined by $T^{\prime \prime}$ as well. According to the literature, the bonding between the particles and the substrate only happens when the impact velocity exceeds some critical values $[6,7]$. The deposition efficiency can increase dramatically with the increasing the impact velocity after the critical value is reached. However, the deposition efficiency is observed to be saturated once the impact velocity reaches certain ceiling value [7]. According to this, it is assumed in this paper that bonding will only happen when $T^{\prime \prime}$ is higher than some critical value and the bonding stress $(\sigma)$ will reach maximum value $\left(\sigma_{\max }\right)$ after reaching ceiling impact speed. To model this assumption mathematically, the following function is proposed:

$$
\sigma=188 \times \frac{1}{1+e^{-1.0 \times 10^{-17} \times\left(T^{\prime \prime}-8.3 \times 10^{18}\right)}}
$$

Song X. et al. (2020) Bonding Strength Improvement ..., In: Itoh S., Shukla S. (eds) Advanced Surface Enhancement. INCASE 2019. Lecture Notes in Mechanical Engineering. Springer, Singapore

https://doi.org/10.1007/978-981-15-0054-1_15 
In this equation, $188.0 \mathrm{MPa}$ is $\sigma_{\max } .1 .0 \times 10^{-17}$ and $8.3 \times 10^{18}$ are some constants which best fit the Ti-6Al-4V data. The fitting can be done automatically using a series of Matlab-driven ABAQUS simulations, where least square fitting was carried out in the Matlab to match experimental data. Table 1 provides the best fitted simulation results with the experimental data.

Table 1. Best-fitted simulation results in comparison with the mini-tensile test data.

\begin{tabular}{ccc}
\hline Impact speed $(\mathrm{m} / \mathrm{s})$ & Simulation result $(\mathrm{MPa})$ & Mini-tensile test $(\mathrm{MPa})$ \\
\hline 650 & 0 & 0 \\
700 & 14 & 18 \\
710 & 34.2 & 28 \\
730 & 65 & 68 \\
760 & 81 & 86 \\
800 & 89 & 90 \\
850 & 103 & 115 \\
\hline
\end{tabular}

Therefore, the local bonding stress can be calculated as a function of its $T$ " value using the equation above and the total bonding force of a single particle can be calculated by integrating the bonding stress over the bonded area. Then the interfacial bonding strength can be obtained by dividing the total force by the average footprint of a single particle in the experiment. Using this equation, we can running single particle impact model at different conditions to predict their bonding strength at the interface.

\section{Conclusion}

In this study, finite element simulation of single particle normal impact process has been carried out to predict the macroscopic coating bonding strength. Temperature secondary jump, which has direct association with the adiabatic shear instability and bonding, has been represented by the value of second order derivative of the temperature history curve. Such value is also associated with the interfacial bonding strength through a Sigmoid function. Such function provides a valuable tool for the metal cold spray community to link the manufacturing process with the coating property directly, which makes the numerical prediction of the bonding strength possible.

\section{References}

1. Assadi, H., Gartner, F., Stoltenhoff, T., Kreye, H.: Bonding Mechanism in Cold Gas Spraying. Acta Materialia 51 (15), 4379-4394 (2003).

Song X. et al. (2020) Bonding Strength Improvement ..., In: Itoh S., Shukla S. (eds) Advanced Surface Enhancement. INCASE 2019. Lecture Notes in Mechanical Engineering. Springer, Singapore

https://doi.org/10.1007/978-981-15-0054-1_15 
2. Song, X., Everaerts, J., Zhai, W., Zheng, H., Tan, W.Y., Sun, W., Li, F., Marinescu, I., Liu, E.J., Korsunsky, A.M.: Residual stresses in single particle splat of metal cold spray process - Numerical simulation and direct measurement, Materials Letters 230, 152-156 (2018).

3. Lesuer, D.: Experimental investigation of material models for Ti-6Al-4V and 2024-T3, Livermore Univ. California, Lawrence Livermore National Laboratory. 1-36 (1999).

4. Tan, W.Y., Sun, W., Phang, Y.P., Dai, M.H., Marinescu, I., Dong, Z.L., Liu, E.J.: Effects of Traverse Scanning Speed of Spray Nozzle on the Microstructure and Mechanical Properties of Cold-Sprayed Ti6A14V Coatings, Journal of Thermal Spray Technology 26 (7), 1484 1497 (2017).

5. Tan, W.Y., Sun, W., Bhowmik, A., Lek, J.Y., Marinescu, I., Li, F., Khun, N.W., Dong, Z.L., Liu, E.J.: Effect of coating thickness on microstructure, mechanical properties and fracture behaviour of cold sprayed Ti6Al4V coatings on Ti6Al4V substrates, Surface \& Coatings Technology 349, 303-317 (2018).

6. Schmidt, T., Gärtner, F., Assadi, H., Kreye, H.: Development of a generalized parameter window for cold spray deposition, Acta Materialia. 54 (3) 729-742 (2006).

7. Schmidt, T., Assadi, H., Gärtner, F., Richter, H., Stoltenhoff, T., Kreye, H., Klassen, T.: From particle acceleration to impact and bonding in cold spraying, Journal of Thermal Spray Technology 18, 794-808 (2009).

Song X. et al. (2020) Bonding Strength Improvement ..., In: Itoh S., Shukla S. (eds) Advanced Surface Enhancement. INCASE 2019. Lecture Notes in Mechanical Engineering. Springer, Singapore

https://doi.org/10.1007/978-981-15-0054-1_15 